\title{
Equilibrio, termodinámica y modelos cinéticos en la adsorción de Rojo 40 sobre tuza de maíz
}

\author{
Danilo Figueroa* \\ Anderson Moreno ${ }^{* *}$ \\ Angelina Hormaza**
}

Recibido: 12/08/2013 • Aceptado: 12/12/2014

\begin{abstract}
Resumen
La adsorción es un método ampliamente utilizado para el tratamiento de contaminantes disueltos. Diversos residuos agroindustriales han sido explorados como potenciales adsorbentes, mostrando alta eficiencia en la eliminación de colorantes. Para el escalado de este proceso se requieren modelos cinéticos y de equilibrio para cada pareja adsorbato-adsorbente. En este trabajo se evaluaron el equilibrio, la cinética y la termodinámica del sistema tuza de maíz-rojo 40 bajo sistema discontinuo a $\mathrm{pH}=2.0$ a las temperaturas de 25,40 y $55^{\circ} \mathrm{C}$. Los modelos de Langmuir, Freundlich y Temkin fueron seleccionados para la representación de las isotermas, en tanto que las ecuaciones de Lagergren, Ho y Elovich, para la cinética del proceso. El modelo de Freundlich presentó el mejor ajuste a las isotermas, la cinética de adsorción fue mejor descrita por la ecuación de Ho y los valores para la energía libre de Gibbs, y la entropía señalaron la espontaneidad y factibilidad del proceso.
\end{abstract}

Palabras clave: isotermas de adsorción, cinética, rojo 40, adsorbentes de bajo costo, tuza de maíz.

\footnotetext{
Ingeniero Químico, Universidad Nacional de Colombia - Sede Medellín. Investigador, Grupo de Investigación SIRYTCOR. Escuela de Química, Facultad de Ciencias, Universidad Nacional de Colombia - Sede Medellín. Dirección: Calle 65 Carrera 64 Autopista Norte, Bloque 15-119. Teléfono: 4309888 extensión 46363. Correo electrónico:dfigueroap@unal.edu.co.

** Ingeniero Químico, Universidad Nacional de Colombia - Sede Medellín. Investigador, Grupo de Investigación SIRYTCOR. Escuela de Química, Facultad de Ciencias, Universidad Nacional de Colombia - Sede Medellín. Dirección: Calle 65 Carrera 64 Autopista Norte, Bloque 15-119. Teléfono: 4309888 extensión 46363. Correo electrónico: amorenosan@unal.edu.co.

*** M. Sc, Ph. D., Profesor Asociado Facultad de Ciencias, Universidad Nacional de Colombia - Sede Medellín. Directora del Grupo de Investigación SIRYTCOR. Escuela de Química, Facultad de Ciencias, Universidad Nacional de Colombia - Sede Medellín. Dirección: Calle 65 Carrera 64 Autopista Norte, Bloque 21-329. Teléfono: 4309851. Correo electrónico: ahormaza@unal.edu.co.
} 


\title{
Equilibrium, thermodynamic and kinetic models for the adsorption of red 40 onto corn cob
}

\begin{abstract}
Adsorption is a widely used method for treatment of dissolved pollutants. Diverse agro-industrial wastes have been explored as potential adsorbents, showing high efficiency in the removal of dyes. To scale this process kinetic and equilibrium models for each pair adsorbate-adsorbent are required. In this work the equilibrium, kinetics and thermodynamic of the corn cob - red 40 system under batch system at pH 2.0 at temperatures of 25, 40 and $55^{\circ} \mathrm{C}$ were evaluated. Langmuir, Freundlich and Temkin models were selected to represent the isotherms, while Lagergren, Ho and Elovich equations for kinetics of the process. Freundlich model provided the best fit to the isotherms, while the adsorption kinetics was best described by the Ho equation and the values for the Gibbs free energy and entropy indicated the spontaneity and feasibility of the process.
\end{abstract}

Keywords: adsorption isotherms, kinetics, red 40, low cost adsorbents, corn cob. 


\section{INTRODUCCIÓN}

La cantidad exacta de colorantes sintéticos producidos en el mundo es desconocida; comercialmente existen más de 100000 variedades y se ha estimado una producción cercana de $7 \times 10^{5}$ a $1 \times 10^{6}$ toneladas por año [1]. Tampoco se conoce la cantidad precisa de tintes descargados en el ambiente, pero se ha reportado que en su proceso de producción, así como en las aplicaciones industriales, un valor aproximado del 10-15 $\%$ es vertido sin tratamiento al ambiente [2].

Los colorantes azo y sus pigmentos son considerados como el grupo químico más grande de los actualmente existentes, alrededor de 26000 tintes. Se ha descubierto mediante pruebas con animales que el grupo azo es un causante de cáncer; por tanto, se prevé su efecto carcinógeno en seres humanos [3]. A este grupo pertenece el colorante rojo 40, (R40), conocido como rojo Allura y comúnmente usado como tinte en alimentos, bebidas y tatuajes [4]. Cantidades mínimas, incluso de $1 \mathrm{ppm}$ de colorante, son suficientes para ocasionar un deterioro estético del medio ambiente [5]. No obstante, la repercusión más drástica de su presencia se verifica en los procesos fotosintéticos, donde una reducción en la penetración de la radiación solar impide los procesos de auto-regeneración del recurso hídrico, afectando la biota acuática [6].

La liberación de cantidades considerables de tintes sintéticos al ambiente ha causado un interés público, problemas de legislación y un serio desafío para los científicos ambientales. Además, la tendencia social por el crecimiento de la protección ambiental y preservación de los recursos naturales promueve el impulso de tecnologías industriales de menor impacto ambiental, que reduzcan el consumo de agua y disminuyan el vertimiento de efluentes altamente coloreados [1].

Dentro de los métodos de tratamiento no tradicionales se encuentra la adsorción, donde se retiene el colorante sobre la superficie de un material adsorbente para obtener un efluente decolorado. El carbón activado es ampliamente usado como adsorbente, pero posee un reducido intervalo de polaridad que impide la remoción de algunos colorantes. Además, puede ser regenerado después de cada ciclo de funcionamiento, pero esto conlleva pérdidas hasta del $10 \%$ del material adsorbente. Por otro lado, su producción y regeneración demandan una gran cantidad de energía, lo que limita su uso en el tratamiento de efluentes coloreados debido a su elevado costo [7-8].

El maíz es uno de los cultivos con mayor producción en el mundo, y genera residuos agrícolas como tuza (TM), hoja y bagazo, los cuales son incinerados en una gran proporción [9]. La TM es, por tanto, un subproducto agrícola renovable, disponible y de bajo costo, para el cual se han reportado capacidades de adsorción de colorantes y metales pesados tanto para el material nativo como para el modificado, que señalan su uso alternativo como potencial adsorbente $[7,10]$. 
El presente trabajo tiene como propósito evaluar la capacidad adsorbente del colorante aniónico R40 sobre TM a través de diferentes isotermas, así como la rapidez del proceso por medio de su cinética, como parámetros fundamentales requeridos para su diseño a mayor escala.

\section{MATERIALES Y MÉTODOS}

\subsection{Preparación del adsorbente}

El residuo agrícola TM fue obtenido en un mercado local de la ciudad. Su preparación incluyó un primer enjuague con agua potable, su secado al aire libre y reducción de su tamaño con un molino de aspas, seleccionando un tamaño de partícula por tamizado entre 0.30 y $0.71 \mathrm{~mm}$. Posteriormente, se lavó con agua destilada y disolución de peróxido de hidrógeno hasta obtener un material libre de material orgánico, se procedió a su secado a una temperatura de $80^{\circ} \mathrm{C}$ durante $24 \mathrm{~h}$ en un horno de convección forzada y finalmente el material fue almacenado herméticamente, controlando periódicamente la humedad con el analizador de humedad marca ADAM.

\subsection{Preparación de la solución de R40 y su cuantificación}

Se utilizó el colorante R40 de tipo comercial obtenido de una industria local. Su solución se preparó en un balón volumétrico de $250 \mathrm{~mL}$ con $250 \mathrm{mg}$ de este tinte y agua desionizada, manteniendo una agitación durante $15 \mathrm{~min}$. La concentración fue determinada mediante espectrofotometría ultravioleta-visible, usando el método lineal con el espectrofotómetro Uv-Vis Perkin-Elmer Lambda 35 a una longitud de onda de máxima absorbancia correspondiente a $\lambda_{\text {máx }}=502 \mathrm{~nm}$. Cabe señalar que previamente se realizó una curva de calibración con concentraciones entre 2.0 y $40 \mathrm{mgL}^{-1}$ de R40 para alcanzar un ajuste de $\mathrm{R}^{2}=0.999$. El pH de la disolución se fijó a un valor de 2.0 con una solución de $\mathrm{HCl}$ de concentración $0.1 \mathrm{~N}$.

\subsection{Ensayos para los estudios termodinámicos}

En un recipiente de vidrio de $100 \mathrm{~mL}$ se depositaron $10 \mathrm{~mL}$ de la solución R40 diluida a una concentración entre 5.0 y $40 \mathrm{mgL}^{-1}$, con una dosificación de $\mathrm{TM}$ de $5.8 \mathrm{gL}^{-1}$, manteniendo una agitación constante de $120 \mathrm{rpm}$. Cada ensayo se llevó a cabo a una temperatura de 25,40 y $55^{\circ} \mathrm{C}$ en un shaker Heidolph provisto de control de temperatura. Una vez transcurridas tres h de duración, se tomó una alícuota, se centrifugó y se midió la concentración al sobrenadante.

\subsection{Ensayos para los estudios cinéticos}

En recipientes de vidrio se tomaron $10 \mathrm{~mL}$ de la solución del colorante $\mathrm{R} 40$ preparada a una concentración de $10 \mathrm{mgL}^{-1}$, con una dosificación de $\mathrm{TM}$ de $5.8 \mathrm{gL}^{-1}$, conservando 
una agitación constante de $120 \mathrm{rpm}$ (estos valores fueron seleccionados como resultado de un diseño de experimentos previo que alcanzó remociones del colorante mayores del $98 \%$ ). Cada ensayo se efectuó a una temperatura de 25,40 y $55^{\circ} \mathrm{C}$, donde las muestras fueron cuantificadas secuencialmente durante un lapso de $150 \mathrm{~min}$. Para cada análisis se prepararon la muestra y dos réplicas, respectivamente, tomando alícuotas de $1.5 \mathrm{~mL}$.

\subsection{Estudios termodinámicos y cinéticos}

El análisis de los datos de adsorción es importante para el desarrollo de ecuaciones de equilibrio y cinética que pueden ser usadas para propósitos de diseño. Al considerarse soluciones diluidas del colorante, se supone que el volumen de solución permanece constante durante el proceso o las pérdidas por evaporación son despreciables; la carga del colorante R40 adsorbido por masa de adsorbente TM se cuantifica mediante la ecuación 1 .

$$
\boldsymbol{q}_{\boldsymbol{t}}=\left(\boldsymbol{C}_{0}-\boldsymbol{C}_{\boldsymbol{t}}\right) \frac{\boldsymbol{V}}{\boldsymbol{W}}
$$

Donde $\mathrm{C}_{0}$ es la concentración inicial de la solución $\left(\mathrm{mgL}^{-1}\right), \mathrm{C}_{\mathrm{t}}$ es la concentración en un instante $\mathrm{t}$ una vez iniciado el proceso, $\mathrm{V}$ es el volumen de la solución (L) y W es la dosificación de TM (g); por tanto, expresamos $\mathrm{q}_{\mathrm{t}}$ como la masa adsorbida de R40 por masa de TM ( $\left.\mathrm{mg} \mathrm{g}^{-1}\right)$ en un instante dado. Si la duración del proceso es lo suficientemente prolongada para alcanzar el equilibrio, tendremos que $q_{t}$ será constante y podemos denominarla $\mathrm{q}_{\mathrm{e}}$, carga en el equilibrio correspondiente a la concentración en el equilibrio $\mathrm{C}_{\mathrm{e}}$, formando la respectiva pareja de datos de equilibrio [11].

\subsubsection{Equilibrio de adsorción}

Los detalles del equilibrio del proceso, generalmente conocidos como isotermas de adsorción, describen el comportamiento de la interacción entre adsorbato-adsorbente y proveen información sobre la capacidad del adsorbente estudiado. Tres modelos, Langmuir, Freundlich y Temkin, cada uno con dos constantes características, son estudiados para este sistema de adsorción R40-TM [11-13].

La isoterma de Langmuir es un modelo teórico válido para la adsorción en una monocapa sobre una superficie completamente homogénea con un número finito de sitios idénticos y específicos de adsorción y con una interacción despreciable entre las moléculas, representado por la ecuación 2.

$$
q_{e}=\frac{q_{\max } K_{L} C_{e}}{1+K_{L} C_{e}}
$$


Donde el parámetro $\mathrm{q}_{\operatorname{máx}}\left(\mathrm{mg} \mathrm{g}^{-1}\right)$ es una constante que denota la capacidad máxima de adsorción, en tanto que la constante $\mathrm{K}_{\mathrm{L}}\left(\mathrm{Lmg}^{-1}\right)$ define la afinidad del adsorbato por el adsorbente.

El modelo de Freundlich es una ecuación empírica que no asume homogeneidad en la energía de los sitios en la superficie y sin límite en la carga máxima de adsorción, y muestra una consistencia de una distribución exponencial de centros activos característicos de una superficie heterogénea, representada como la ecuación 3.

$$
q_{e}=K_{F} C_{e}^{1 / n}
$$

Los parámetros $\mathrm{K}_{\mathrm{F}}\left(\mathrm{mg} \mathrm{g}^{-1}\left(\mathrm{mgL}^{-1}\right)^{-1 / n}\right)$ y $\mathrm{n}$ caracterizan la capacidad y la intensidad de adsorción, respectivamente; hipotéticamente $\mathrm{K}_{\mathrm{F}}$ alcanza el valor de $\mathrm{q}_{\mathrm{e}}$ cuando la $\mathrm{C}_{\mathrm{e}}$ se aproxima a la unidad y así puede ser considerado como un indicador de la intensidad de adsorción.

El Modelo de Temkin, caracterizado por una distribución uniforme de energía de unión, hasta una cierta máxima energía de unión, introduce las constantes cuyos valores dependen del calor de adsorción inicial y supone una disminución lineal del calor de adsorción con el grado de recubrimiento, expresada en la ecuación 4.

$$
\boldsymbol{q}_{e}=\boldsymbol{B} \ln \left(\boldsymbol{A} \boldsymbol{C}_{e}\right)
$$

Donde B es una constante que está relacionada con el calor de adsorción y A ( $\left.\mathrm{Lmg}^{-1}\right)$ es la constante de unión en equilibrio correspondiente a la máxima energía de unión.

\subsubsection{Parámetros termodinámicos}

Los parámetros termodinámicos reflejan la factibilidad y la naturaleza espontánea del proceso; así, el cambio de energía libre, el cambio de entalpía y el cambio de entropía de superficie pueden ser estimados usando el cambio de la constante de equilibrio con la temperatura absoluta. El equilibrio heterogéneo que se alcanza puede ser representado con la constante aparente de equilibrio $\mathrm{K}_{\mathrm{c}}^{\prime}$ definida mediante la ecuación 5.

$$
\boldsymbol{K}_{c}^{\prime}=\frac{\boldsymbol{C}_{a d, e}}{\boldsymbol{C}_{e}}
$$

Donde $\mathrm{C}_{\mathrm{ad}, \mathrm{e}}$ es la concentración del colorante adsorbido sobre el adsorbente, $\mathrm{y}$ al considerar la dosis de adsorbente en $\mathrm{gL}^{-1}$, el valor de $\mathrm{C}_{\mathrm{ad}, \mathrm{e}}$ será el valor de $\mathrm{q}_{\mathrm{e}}$, se obtienen diferentes valores de la constante aparente por cada pareja de datos pero se seleccionan aquellos valores correspondientes a las concentraciones más diluidas. Hipotéticamente extrapolando la concentración a cero, el valor de la constante apa- 
rente se tomará como la constante de equilibrio estándar $\mathrm{K}_{\mathrm{c}}^{0}$ del sistema de adsorción; particularmente con la isoterma de Langmuir se puede calcular $\mathrm{K}_{\mathrm{c}}^{0} \operatorname{como} \mathrm{q}_{\max } * \mathrm{~K}_{\mathrm{L}}$. Con el valor de $\mathrm{K}^{0}{ }_{\mathrm{c}}$ se calcula el cambio en la energía libre de adsorción $\Delta \mathrm{G}^{\circ}\left(\mathrm{kJ}^{\mathrm{mol}}{ }^{-1}\right)$ en la respectiva temperatura. Donde $\mathrm{R}$ es la constante universal de los gases (8.314 J $\mathrm{mol}^{-1} \mathrm{~K}^{-1}$ ) y $\mathrm{T}(\mathrm{K})$ la temperatura absoluta. El cambio en la energía libre indica el grado de espontaneidad del proceso; valores más negativos reflejan una mayor favorabilidad de la adsorción y su variación puede expresarse en función del cambio en la entalpía $\Delta \mathrm{H}^{\circ}\left(\mathrm{kJ} \mathrm{mol}^{-1}\right)$ y la entropía $\Delta \mathrm{S}^{\circ}\left(\mathrm{J} \mathrm{mol}^{-1} \mathrm{~K}^{-1}\right)$ de adsorción con la temperatura, obteniéndose la recta de regresión con pendiente $-\Delta \mathrm{S}^{\circ}$ e intercepto $\Delta \mathrm{H}^{\circ}$, tal como muestra la ecuación 7.

$$
\Delta G^{\circ}=\Delta H^{\circ}-T \Delta S^{\circ}
$$

Un valor negativo de $\Delta \mathrm{H}^{\circ}$ refleja que el proceso es de carácter exotérmico, mientras que valores negativos de $\Delta \mathrm{S}^{\circ}$ indican una disminución de la aleatoriedad en la interfase solución-sólido durante la adsorción [2,14].

\subsubsection{Cinética de adsorción}

En la adsorción, como proceso dependiente del tiempo, es necesario conocer su rapidez para el diseño y evaluación de adsorbentes. Para representar los modelos cinéticos de adsorción se emplean dos criterios: la concentración de la solución o la carga en el adsorbente. Generalmente, los modelos de Lagergren de pseudo primer orden, Ho pseudo segundo orden y Elovich han sido comúnmente usados como modelos simplificados en la dinámica de adsorción [2,9,11].

El modelo de Lagergren de pseudo primer orden está descrito matemáticamente por la ecuación 8 .

$$
\frac{d q_{t}}{d t}=K_{1}\left(q_{e}-q_{t}\right)
$$

Donde la $\mathrm{K}_{1}\left(\mathrm{~min}^{-1}\right)$ es la constante cinética de adsorción de primer orden y $\mathrm{q}_{\mathrm{e}}$ es la carga de colorante en el equilibrio, donde este parámetro debe ser establecido previamente antes de determinar el ajuste del modelo, considerando al adsorbente inicialmente libre de soluto, es decir, bajo las condiciones de contorno $t=0$ con $q_{t}=0$ $\mathrm{yt}=\mathrm{t}$ con $\mathrm{q}_{\mathrm{t}}=\mathrm{q}_{\mathrm{t}}$, de forma integrada tenemos la ecuación 9 .

$$
q_{t}=q_{e}\left(1-e^{-K_{1} t}\right)
$$


Para el modelo de Ho de pseudo segundo orden, usualmente se atribuye este modelo al proceso que envuelve un mecanismo de quimiadsorción; es de destacar su particularidad para estimar la carga en equilibrio, tal como se muestra en la ecuación 10.

$$
\frac{d q_{t}}{d t}=K_{2}\left(q_{e}-q_{t}\right)^{2}
$$

Donde $\mathrm{K}_{2}\left(\mathrm{~g} \mathrm{mg}^{-1} \mathrm{~min}^{-1}\right)$ es la constante cinética de adsorción de segundo orden, cuya integración bajo las mismas condiciones de contorno, previamente descritas, se representa mediante la ecuación 11.

$$
\boldsymbol{q}_{\boldsymbol{t}}=\frac{\boldsymbol{K}_{2} \boldsymbol{q}_{e}^{2} \boldsymbol{t}}{1+\boldsymbol{K}_{2} \boldsymbol{q}_{e} \boldsymbol{t}}
$$

El modelo de Elovich, de aplicación general en procesos de quimiadsorción, supone que los sitios activos del adsorbente son heterogéneos y por ello exhiben diferentes energías de activación, basándose en un mecanismo de reacción de segundo orden para un proceso de reacción heterogénea, descrito matemáticamente en la ecuación 12 .

$$
\frac{d q_{t}}{d t}=\alpha e^{-\beta q_{t}}
$$

Donde $\alpha\left(\mathrm{mg} \mathrm{g}^{-1} \min ^{-1}\right)$ es la velocidad inicial de adsorción y $\beta$ está relacionada con la superficie cubierta y energía de activación de quimiadsorción; se representa en la ecuación 13.

$$
q_{t}=\frac{1}{\beta} \ln (\beta \alpha t)
$$

\subsubsection{Energía de activación}

La energía de activación de adsorción $\mathrm{E}_{\mathrm{a}}\left(\mathrm{kJ} \mathrm{mol}^{-1}\right)$ fue calculada mediante la ecuación 14, en la forma linealizada de Arrhenius:

$$
\ln \boldsymbol{K}=\ln \boldsymbol{A}-\left(\boldsymbol{E}_{a} / \boldsymbol{R} \boldsymbol{T}\right)
$$

Donde el valor $\mathrm{K}$ es la constante cinética que controla el proceso a la temperatura absoluta T (K); además, esta expresión permite calcular la constante de Arrhenius A.

\subsection{ANÁLISIS DE ERROR}


Con el propósito de encontrar el modelo que mejor se ajuste a los datos experimentales se consideraron el coeficiente de determinación $\mathrm{R}^{2}$ y 4 errores estadísticos, el porcentaje de error promedio estándar $\varepsilon(\%)$, chi-cuadrada $\chi_{i}^{2}$, suma de errores cuadrados SSE y suma errores absolutos SAE, descritos matemáticamente en las ecuaciones 15, 16, 17 y 18 , respectivamente.

$$
\begin{gathered}
\varepsilon(\%)=\frac{1}{n} \sum\left|\frac{y_{c}-y_{e}}{y_{e}}\right| \\
\chi_{i}^{2}=\sum \frac{\left(y_{e}-y_{c}\right)^{2}}{y_{c}} \\
S S E=\sum\left(y_{e}-y_{c}\right)^{2} \\
S A E=\sum\left|\left(y_{e}-y_{c}\right)\right|
\end{gathered}
$$

El ajuste de los modelos se realizó mediante regresión no-lineal en la minimización de $\chi_{\mathrm{i}}^{2}$, donde $y_{\mathrm{e}}$ es el valor de experimental y $y_{\mathrm{c}}$ corresponde al valor calculado con la ecuación matemática. La selección del modelo más adecuado se basó en los criterios de mejor ajuste y menor error registrado. Para el procesamiento de los datos se utilizó el programa de Microsoft Office Excel y el complemento de optimización Solver $[14,15]$.

\section{RESULTADOS}

\subsection{Estudios de equilibrio, cinética y termodinámica}

La representación gráfica de los modelos de equilibrio para TM-R40 se observa en las figuras $1 \mathrm{a}, 1 \mathrm{~b}$ y $1 \mathrm{c}$ a las temperaturas de $25{ }^{\circ} \mathrm{C}, 40^{\circ} \mathrm{C}$ y $55^{\circ} \mathrm{C}$. En el intervalo de concentración estudiado se aprecia un comportamiento creciente de la carga adsorbida y una curva cóncava hacia el eje de la concentración, característico del tipo L en la clasificación de isotermas propuesta por Giles [16], donde, además, no es evidente un límite de adsorción máxima. 

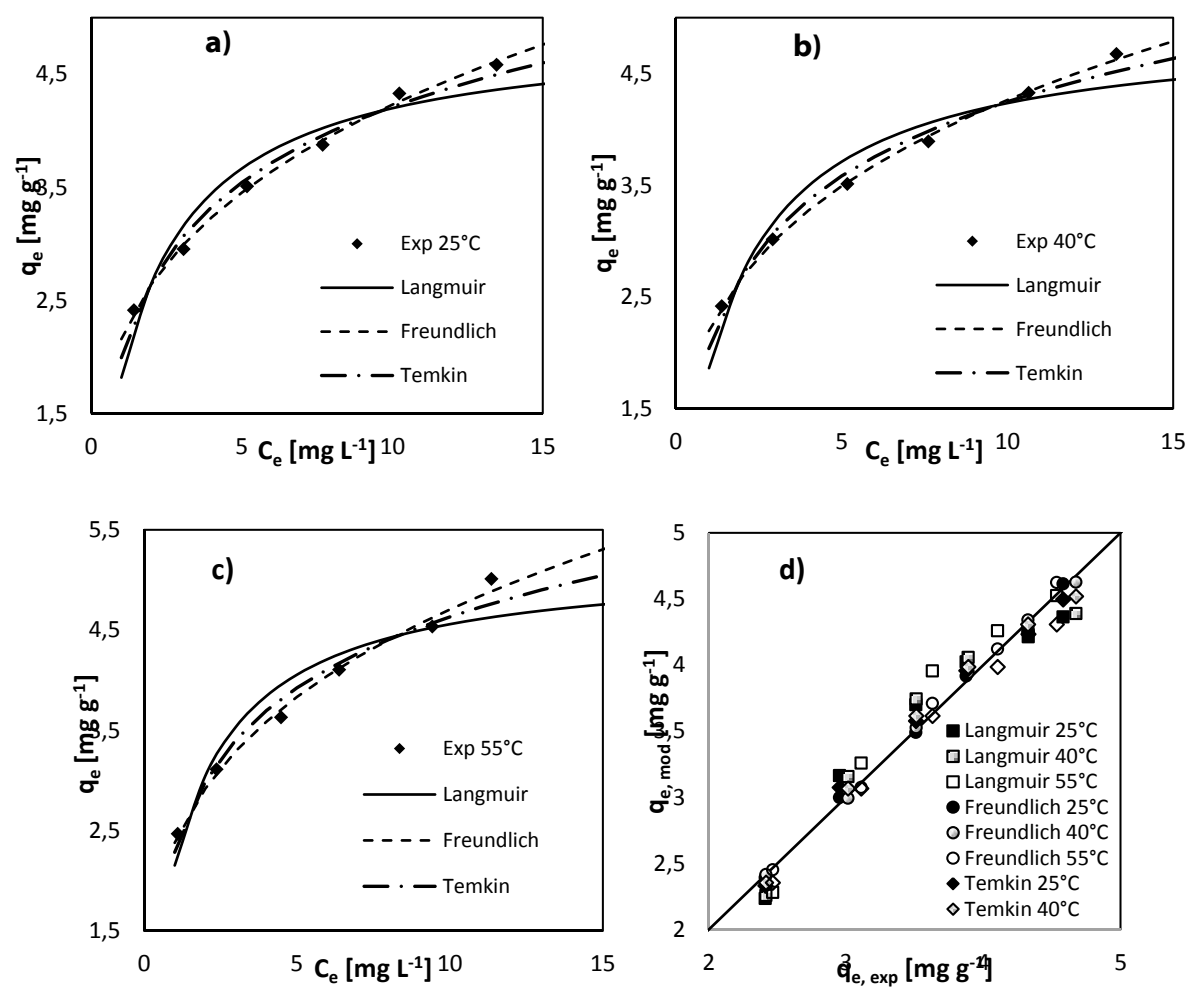

Figura 1. Representación de los modelos ajustados a la temperatura a) $25^{\circ} \mathrm{C}$, b) $\left.40^{\circ} \mathrm{C}, \mathrm{c}\right) 55^{\circ} \mathrm{C}$ y d) comparación de datos experimentales y teóricos

Fuente: elaboración propia

La comparación de los valores estimados con los experimentales, (figura 1d) muestra la magnitud de la desviación de cada modelo a medida que se aleja de la recta; se observa que el modelo de Langmuir presenta la mayor desviación, al sobrestimar la carga en las concentraciones medias, y subestimarla a concentraciones altas; un efecto similar se registra para el modelo de Temkin, pero con una desviación mucho menor, en tanto que el modelo de Freundlich ofrece el mejor ajuste a los datos experimentales en todas las temperaturas y concentraciones analizadas.

Los parámetros de cada modelo de isoterma se presentan en la tabla 1. El análisis de error señala a la isoterma de Freundlich como el modelo más adecuado en la representación del equilibrio del proceso de adsorción al poseer excelentes correlaciones $\left(\mathrm{R}^{2}>0.99\right)$ y bajos valores en el porcentaje de error promedio estándar $(\varepsilon<1.5 \%)$ en todas las temperaturas evaluadas. Asimismo, el modelo Temkin, con correlaciones $\mathrm{R}^{2}$ mayores de $0.97 \mathrm{y}$ errores $\varepsilon$ menores del $3.0 \%$, puede representar de forma aceptable 
las isotermas. Por el contrario, Langmuir obtuvo la más baja correlación y el mayor error, descartándolo como modelo ajustable para el equilibrio del sistema TM-R40.

Tabla 1. Parámetros de los modelos ajustados de isotermas a) Langmuir, b) Freundlich, c) Temkin y d) Parámetros termodinámicos

\begin{tabular}{|c|c|c|c|c|c|c|c|}
\hline \multicolumn{8}{|c|}{ a) LANGMUIR } \\
\hline $\mathbf{T}[\mathrm{K}]$ & $\mathbf{q}_{\max }\left[\mathrm{mg} \mathrm{g}^{-1}\right]$ & $\mathrm{K}_{\mathrm{L}}$ & $\mathbf{R}^{2}$ & $\chi_{i}^{2}$ & $\varepsilon(\%)$ & SSE & SAE \\
\hline 298 & 4.913 & 0.587 & 0.9432 & 0.0571 & 5.1743 & 0.1937 & 1.0565 \\
\hline 313 & 4.933 & 0.607 & 0.9400 & 0.0580 & 4.9149 & 0.2107 & 1.0370 \\
\hline 328 & 5.205 & 0.705 & 0.9244 & 0.0857 & 5.4766 & 0.3311 & 1.2052 \\
\hline \multicolumn{8}{|c|}{ b) FREUNDLICH } \\
\hline $\mathrm{T}[\mathrm{K}]$ & $\mathrm{K}_{\mathrm{F}}$ & $\mathrm{n}$ & $\mathbf{R}^{2}$ & $\chi_{i}^{2}$ & $\varepsilon(\%)$ & SSE & SAE \\
\hline 298 & 2.159 & 3.425 & 0.9970 & 0.0027 & 1.0283 & 0.0101 & 0.2233 \\
\hline 313 & 2.197 & 3.474 & 0.9983 & 0.0015 & 0.6639 & 0.0059 & 0.1539 \\
\hline 328 & 2.379 & 3.378 & 0.9929 & 0.0071 & 1.4712 & 0.0313 & 0.3600 \\
\hline \multicolumn{8}{|c|}{ c) TEMKIN } \\
\hline $\mathrm{T}[\mathrm{K}]$ & A & B & $\mathbf{R}^{2}$ & $\chi_{i}^{2}$ & $\varepsilon(\%)$ & SSE & SAE \\
\hline 298 & 7.929 & 0.963 & 0.9857 & 0.0143 & 2.5891 & 0.0487 & 0.5311 \\
\hline 313 & 8.371 & 0.959 & 0.9855 & 0.0133 & 2.2573 & 0.0511 & 0.4896 \\
\hline 328 & 9.390 & 1.020 & 0.9748 & 0.0268 & 2.9161 & 0.1104 & 0.6714 \\
\hline \multicolumn{8}{|c|}{ d) Parámetros termodinámicos } \\
\hline \multicolumn{3}{|c|}{$\Delta \mathrm{G}^{\circ}\left(\mathbf{k J} \mathbf{~ m o l}^{-1}\right)$} & \multirow{2}{*}{\multicolumn{2}{|c|}{$\begin{array}{c}\Delta \mathrm{H}^{\circ} \\
\left(\mathbf{k J} \mathbf{~ m o l}^{-1}\right)\end{array}$}} & \multirow{2}{*}{\multicolumn{2}{|c|}{$\begin{array}{c}\Delta \mathrm{S}^{\circ} \\
(\mathrm{J} \mathrm{mol} \\
\left.\mathbf{l}^{-1} \mathrm{~K}^{-1}\right)\end{array}$}} & \multirow{2}{*}{$\mathbf{R}^{2}$} \\
\hline$T[K] 298$ & 313 & 328 & & & & & \\
\hline-2.63 & -2.85 & -3.54 & \multicolumn{2}{|c|}{6.58} & \multicolumn{2}{|c|}{30.65} & 0.922 \\
\hline
\end{tabular}

Fuente: elaboración propia

Los valores de $\Delta G^{\circ}$ fueron negativos y muestran un incremento en su magnitud con el ascenso de la temperatura. Se alcanzó una correlación $\mathrm{R}^{2}$ de 0.92 para la regresión de los parámetros termodinámicos con la ecuación 17 , y se encontraron valores

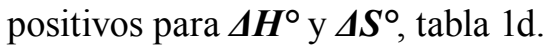

Con respecto a la cinética de adsorción, se encontró para las tres temperaturas un mejor ajuste con el modelo de Ho pseudo segundo orden, al obtener correlaciones 
cercanas a 0.93 y errores tan bajos $\varepsilon$ menores del $3.0 \%$, tabla 2 . El valor de la energía de activación, $\mathrm{E}_{\mathrm{a}}=8.197 \mathrm{~kJ} \mathrm{~mol}^{-1}$, fue determinado a partir de la constante de velocidad de segundo orden K2, mediante la ecuación 14; su valor positivo corresponde al carácter esperado para este proceso [16].

Tabla 2. Parámetros de los modelos cinéticos a) Lagergren pseudo primer orden b) Ho pseudo segundo orden c) Elovich y d) Energía de activación

\begin{tabular}{|c|c|c|c|c|c|c|c|}
\hline \multicolumn{8}{|c|}{ a) LAGERGREN } \\
\hline $\mathrm{T}[\mathrm{K}]$ & $\mathrm{K}_{1}\left[\mathrm{~min}^{-1}\right]$ & $\mathrm{q}_{\mathrm{e}}\left[\mathrm{mg} \mathrm{g}^{-1}\right]$ & $\mathrm{R}^{2}$ & $\chi_{\mathrm{i}}^{2}$ & $\varepsilon(\%)$ & SSE & SAE \\
\hline 298 & 0.872 & 1.622 & 0.7017 & 0.0872 & 6.4653 & 0.1263 & 1.0301 \\
\hline 313 & 0.987 & 1.616 & 0.7242 & 0.0635 & 5.1755 & 0.0949 & 0.8320 \\
\hline 328 & 1.256 & 1.736 & 0.5538 & 0.0843 & 5.7953 & 0.1433 & 1.0342 \\
\hline \multicolumn{8}{|c|}{ b) $\mathrm{HO}$} \\
\hline $\mathrm{T}[\mathrm{K}]$ & $\begin{array}{c}\mathrm{K}_{2}\left[\mathrm{~g} \mathrm{mg}^{-1}\right. \\
\left.\mathrm{min}^{-1}\right]\end{array}$ & $\mathrm{q}_{\mathrm{e}}\left[\mathrm{mg} \mathrm{g}^{-1}\right]$ & $\mathbf{R}^{2}$ & $\chi_{\mathrm{i}}^{2}$ & $\varepsilon(\%)$ & SSE & SAE \\
\hline 298 & 0.817 & 1.707 & 0.9544 & 0.0150 & 2.4379 & 0.0193 & 0.3720 \\
\hline 313 & 0.968 & 1.693 & 0.9651 & 0.0091 & 1.7873 & 0.0120 & 0.2737 \\
\hline 328 & 1.105 & 1.813 & 0.8835 & 0.0248 & 3.0049 & 0.0374 & 0.5154 \\
\hline \multicolumn{8}{|c|}{ c) ELOVICH } \\
\hline $\mathbf{T}[\mathbf{K}]$ & $\beta$ & $\alpha\left[\mathrm{mg} \mathrm{g}^{-1} \mathbf{m i n}\right]$ & $\mathbf{R}^{2}$ & $\chi_{\mathrm{i}}^{2}$ & $\varepsilon(\%)$ & SSE & SAE \\
\hline 298 & 7.732 & 1415.269 & 0.8575 & 0.0409 & 4.4392 & 0.0603 & 0.7261 \\
\hline 313 & 8.698 & 5855.052 & 0.8335 & 0.0392 & 4.3561 & 0.0573 & 0.7094 \\
\hline 328 & 7.970 & 5855.052 & 0.8652 & 0.0248 & 3.2316 & 0.0433 & 0.6102 \\
\hline \multicolumn{8}{|c|}{ d) Energía de activación } \\
\hline \multicolumn{3}{|c|}{$\mathrm{K}_{2}\left[\mathrm{~kJ} \mathrm{~mol}^{-1}\right]$} & & \multirow{2}{*}{\multicolumn{2}{|c|}{$\begin{array}{c}\mathrm{Ea} \\
{\left[\mathrm{kJ} \mathrm{mol}^{-1}\right]}\end{array}$}} & \multirow{2}{*}{\multicolumn{2}{|c|}{ A }} \\
\hline $\mathrm{T}[\mathrm{K}] 298$ & 313 & 328 & & & & & \\
\hline 0.817 & 0.968 & 1.105 & & \multicolumn{2}{|c|}{8.197} & \multicolumn{2}{|c|}{22.414} \\
\hline
\end{tabular}

Fuente: elaboración propia

Las gráficas de los modelos cinéticos para el sistema TM-R40 se presentan en las figuras $2 \mathrm{a}, 2 \mathrm{~b}$ y $2 \mathrm{c}$ para cada una de las temperaturas analizadas. Se observa que la curva del modelo de Ho pseudo segundo orden se ajusta muy bien a los datos experimentales, lo que confirma lo obtenido por el análisis de error. Los otros modelos 
presentan sobre y subestimaciones que reflejan serias desviaciones. En general, cabe resaltar la rápida ocurrencia del proceso, pues alrededor de los 15 minutos se alcanza al menos el $90 \%$ de toda la carga en el equilibrio, mientras que el valor restante puede requerir hasta 60 minutos.
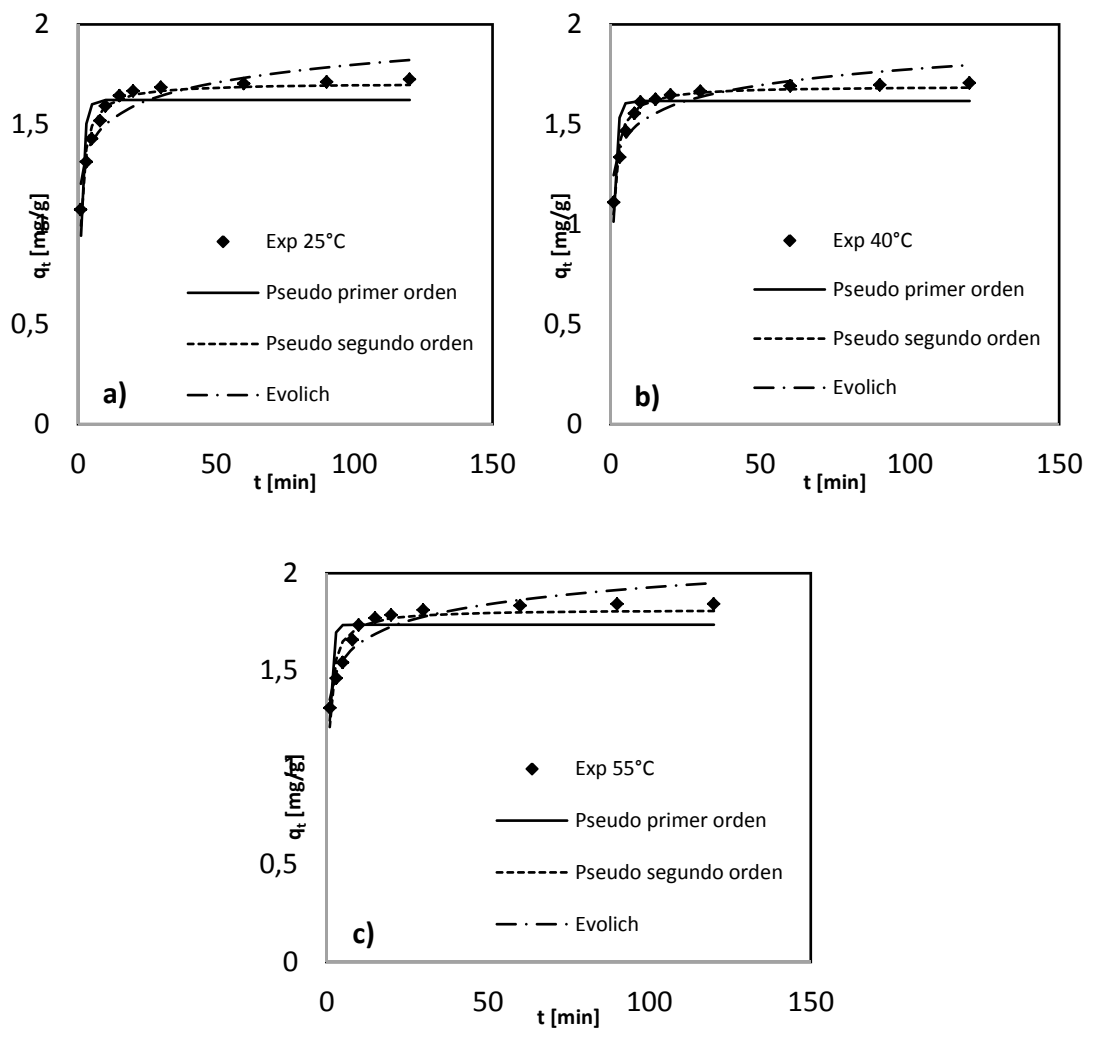

Figura 2. Modelos de la cinética de segundo orden del sistema TM-R40 ajustados a la temperatura de a) $25^{\circ} \mathrm{C}$, b) $40^{\circ} \mathrm{C}$ y c) $55^{\circ} \mathrm{C}$

Fuente: elaboración propia

\section{DISCUSIÓN}

Para el equilibrio, al incrementar la temperatura se observó un aumento en la carga adsorbida a la respectiva concentración, siendo esta diferencia más acentuada al pasar de 40 a $55^{\circ} \mathrm{C}$. Este efecto es mejor representado en la magnitud del parámetro $\mathrm{K}_{\mathrm{F}}$, el cual señala de manera relativa la intensidad de adsorción; se aprecia que un aumento de la temperatura conduce a un incremento en el valor de esta constante y, por tanto, una 
mayor carga del colorante en el adsorbente, especialmente a una concentración determinada. Resultados similares son descritos para colorantes de naturaleza aniónica [17].

Por otro lado, el valor de $\mathrm{n}$ es mucho mayor a la unidad en todas las temperaturas evaluadas, característico de curvas cóncavas hacia el eje de la concentración, mostrando la favorabilidad del proceso. Además, el comportamiento creciente en la isoterma sugiere que la adsorción ocurre en multicapas sin un límite aparente de adsorción máxima; no obstante, al usar el modelo Freundlich para extrapolar una supuesta carga del colorante a una concentración de R40 de $1.0 \mathrm{gL}^{-1}$, esta carga sería de $18 \mathrm{mg} \mathrm{g}^{-1}$, ofreciendo un indicio de la carga esperada a altas concentraciones.

El parámetro A, correspondiente a la máxima energía de unión del modelo de Temkin, presenta un incremento notable con el aumento de la temperatura, mientras que el valor del parámetro $\mathrm{B}$, que señala el calor de adsorción, no muestra una tendencia clara, pero posee el más alto valor a la mayor temperatura evaluada de $55^{\circ} \mathrm{C}$. Estos dos comportamientos sugieren la naturaleza endotérmica del proceso de adsorción [18]. Los valores negativos obtenidos para $\Delta \mathrm{G}^{\circ}$ confirman la factibilidad del proceso y su naturaleza espontánea. Por el contrario, el valor del cambio de entalpía, $\Delta \mathrm{H}^{\circ}$, fue positivo, señalando el carácter endotérmico del proceso. Por otro lado, el valor positivo de $\Delta \mathrm{S}^{\circ}$ refleja la afinidad del adsorbente por el colorante en solución y algunos cambios estructurales en el sorbato y adsorbente [2,9].

Por su parte, el modelo de Ho pseudo segundo orden mostró un buen ajuste y señaló que la capacidad de adsorción estimada crece con un aumento de la temperatura, aspecto que concuerda con lo explicado mediante el estudio de isotermas. La cinética obtenida para el proceso es comparable a la de otros colorantes ácidos, tanto por el valor de las constantes como por su ocurrencia en los primeros 15 minutos con el $90 \%$ de la carga de equilibrio [19].

En particular, el valor de la constante $\mathrm{K}_{2}$ presenta un aumento con el incremento de la temperatura, señalando que la velocidad del proceso se ve favorecida a medida que la temperatura asciende. El valor obtenido para la energía de activación $\left(\mathrm{E}_{\mathrm{a}}=8.197 \mathrm{~kJ}\right.$ $\mathrm{mol}^{-1}$ ), se encuentra en el rango establecido para un proceso que tiene lugar mediante una fisiadsorción [9,20], naturaleza que concuerda con el criterio de la cinética de pseudo segundo orden, con la cual se encontró la mejor correlación para el presente estudio.

\section{CONCLUSIONES}

El equilibrio del proceso de adsorción de la pareja TM-R40 es mejor descrito por el modelo de Freundlich, donde las isotermas con su comportamiento creciente señalan la formación de multicapas sobre el adsorbente, propio de sistemas de adsorción de 
tipo físico. Por su parte, la cinética es mejor representada por el modelo de Ho pseudo segundo orden, donde, además, su rápida ocurrencia ofrece ventajas para posteriores desarrollos de escalado. Finalmente, el valor negativo para la energía libre de Gibbs de superficie y el valor positivo para la entropía señalan la favorabilidad y espontaneidad del proceso que debido a su carácter endotérmico se ve favorecido con el aumento de la temperatura. En definitiva, la pareja TM-R40 constituye un sistema adecuado y factible de escalar, pues el proceso de adsorción es espontáneo, endotérmico con un equilibrio y cinética favorecidos a altas temperaturas.

\section{AGRADECIMIENTOS}

Los autores expresan agradecimientos a la Universidad Nacional de Colombia - Sede Medellín por el apoyo para la infraestructura del Laboratorio de Química Experimental; asimismo, al Programa Jóvenes Investigadores e Innovadores Año 2013, Convocatoria 617, por el respaldo financiero del Proyecto código 201010013080 y a la Vicerrectoría de Investigación - Dirección Nacional de Extensión por el financiamiento del proyecto GTI Código 40000001.

\section{REFERENCIAS}

[1] E. Forgacs, T. Cserháti, and G. Oros, "Removal of synthetic dyes from wastewaters: a review", Environment International, vol. 30, n. ${ }^{\circ}$, pp. 953-71, 2004.

[2] Z. Aksu, A. İ. Tatlı, and Ö. Tunç, “A comparative adsorption/biosorption study of Acid Blue 161: Effect of temperature on equilibrium and kinetic parameters", Chemical Engineering Journal, vol. 142, n. ${ }^{\circ}$, pp. 23-39, 2008.

[3] P. Tripathi, V. C. Srivastava, and A. Kumar, "Optimization of an azo dye batch adsorption parameters using Box-Behnken design” Desalination, vol. 249, n. ${ }^{\circ}$ 3, pp. 1273-1279, 2009.

[4] M. Soylak, Y. E. Unsal, and M. Tuzen, "Spectrophotometric determination of trace levels of allura red in water samples after separation and preconcentration", Food and chemical toxicology: an international journal published for the British Industrial Biological Research Association, vol. 49, n. ${ }^{\circ}$, pp. 1183-7, 2011.

[5] G. Crini, "Non-conventional low-cost adsorbents for dye removal: a review", Bioresource technology, vol. 97, n. ${ }^{\circ}$, pp. 1061-85, 2006.

[6] D. Brown, "Effects of colorants in the aquatic environment", Ecotoxicology and Environmental Safety, vol. 13, n. ${ }^{\circ}$, pp. 139-147, 1987.

[7] V. K. Gupta and Suhas, "Application of low-cost adsorbents for dye removal--a review", Journal of Environmental Management, vol. 90, n. ${ }^{\circ}$ 8, pp. 2313-42, 2009.

[8] T. Robinson, G. McMullan, R. Marchant, and P. Nigam, "Remediation of dyes in textile effluent: 
a critical review on current treatment technologies with a proposed alternative", Bioresource technology, vol. 77, n. ${ }^{\circ}$, pp. 247-55, 2001.

[9] L. Zheng, Z. Dang, X. Yi, and H. Zhang, "Equilibrium and kinetic studies of adsorption of $\mathrm{Cd}(\mathrm{II})$ from aqueous solution using modified corn stalk", Journal of Hazardous Materials, vol. 176, n. ${ }^{\circ} 1-3$, pp. 650-6, 2010.

[10] P. Miretzky and a F. Cirelli, “Cr(VI) and $\mathrm{Cr}$ (III) removal from aqueous solution by raw and modified lignocellulosic materials: a review”, Journal of Hazardous Materials, vol. 180, n. ${ }^{\circ}$ 1-3, pp. 1-19, 2010.

[11] V. Vadivelan and K. V. Kumar, "Equilibrium, kinetics, mechanism, and process design for the sorption of methylene blue onto rice husk", Journal of colloid and interface science, vol. 286, n. ${ }^{\circ} 1$, pp. 90-100, 2005.

[12] Y.-S. Ho, W.-T. Chiu, and C.-C. Wang, "Regression analysis for the sorption isotherms of basic dyes on sugarcane dust”, Bioresource technology, vol. 96, n. ${ }^{\circ}$ 11, pp. 1285-91, 2005.

[13] I. a W. Tan, a L. Ahmad, and B. H. Hameed, "Adsorption isotherms, kinetics, thermodynamics and desorption studies of 2, 4, 6-trichlorophenol on oil palm empty fruit bunch-based activated carbon", Journal of Hazardous Materials, vol. 164, n. ${ }^{\circ}$ 2-3, pp. 473-82, 2009.

[14] Z. Aksu and I. A. Isoglu, "Use of agricultural waste sugar beet pulp for the removal of Gemazol turquoise blue-G reactive dye from aqueous solution", Journal of Hazardous Materials, vol. 137, n. ${ }^{\circ}$ 1, pp. 418-30, 2006.

[15] R. Han, Y. Wang, W. Zou, Y. Wang, and J. Shi, "Comparison of linear and nonlinear analysis in estimating the Thomas model parameters for methylene blue adsorption onto natural zeolite in fixed-bed column", Journal of Hazardous Materials, vol. 145, n. ${ }^{\circ} 1-2$, pp. 331-5, 2007.

[16] G. Limousin, J.-P. Gaudet, L. Charlet, S. Szenknect, V. Barthès, and M. Krimissa, "Sorption isotherms: A review on physical bases, modeling and measurement", Applied Geochemistry, vol. 22, n. ${ }^{\circ}$ 2, pp. 249-275, 2007.

[17] L. Yu, Y.-M. Luo. "The adsorption mechanism of anionic and cationic dyes by Jerusalem artichoke stalk-based mesoporous activated carbon", Journal of Environmental Chemical Engineering, vol. 2, pp. 220-229, 2014.

[18] M. A. Malana, S. Ijaz, y M. N. Ashiq, "Removal of various dyes from aqueous media onto polymeric gels by adsorption process: Their kinetics and thermodynamics", Desalination, vol. 263, n. ${ }^{\circ} 1-3$, pp. 249-257, 2010.

[19] M. Arami, N. Y. Limaee, N. M. Mahmoodi, y N. S. Tabrizi, "Equilibrium and kinetics studies for the adsorption of direct and acid dyes from aqueous solution by soy meal hull", Journal of Hazardous Materials, vol. 135, n. ${ }^{\circ}$ 1-3, pp. 171-9, 2006.

[20] H. Nollet, M. Roels, P. Lutgen, P. Van Der Meeren y W. Verstraete. "Removal of PCBs from wastewater using fly ash”, Chemosphere, 53(6), pp. 655-65, 2003. 